OPEN ACCESS

Edited by: Hari S. Misra,

Bhabha Atomic Research Centre (BARC), India

Reviewed by: Vijay Pancholi,

The Ohio State University, United States

Yen-Ping Hsueh,

Academia Sinica, Taiwan

Gisele LaPointe,

University of Guelph, Canada

*Correspondence: Jinkui Yang

jinkui960@ynu.edu.cn

Specialty section:

This article was submitted to Microbial Physiology and Metabolism,

a section of the journal Frontiers in Microbiology

Received: 05 January 2021 Accepted: 09 April 2021

Published: 22 June 2021

Citation:

Xie M, Yang J, Jiang K, Bai N, Zhu M, Zhu Y, Zhang K-Q and Yang J (2021) AoBck1 and AoMkk1 Are Necessary to Maintain Cell Wall Integrity, Vegetative Growth, Conidiation, Stress Resistance, and Pathogenicity in the Nematode-Trapping Fungus Arthrobotrys oligospora.

Front. Microbiol. 12:649582. doi: 10.3389/fmicb.2021.649582

\section{AoBck1 and AoMkk1 Are Necessary to Maintain Cell Wall Integrity, Vegetative Growth, Conidiation, Stress Resistance, and Pathogenicity in the Nematode-Trapping Fungus Arthrobotrys oligospora}

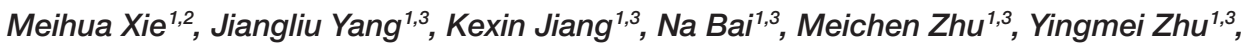 \\ Ke-Qin Zhang ${ }^{1,3}$ and Jinkui Yang ${ }^{1,3 *}$
}

\begin{abstract}
'State Key Laboratory for Conservation and Utilization of Bio-Resources in Yunnan, and Key Laboratory for Southwest Microbial Diversity of the Ministry of Education, Yunnan University, Kunming, China, ${ }^{2}$ School of Resources, Environment and Chemistry, Chuxiong Normal University, Chuxiong, China, ${ }^{3}$ School of Life Science, Yunnan University, Kunming, China
\end{abstract}

The cell wall integrity (CWI) pathway is composed of three mitogen-activated protein kinases (MAPKs), Bck1, Mkk1/2, and Slt2, and is one of the main signaling pathways for fungal pathogenesis, cell wall synthesis, and integrity maintenance. In this study, we characterized orthologs of Saccharomyces cerevisiae Bck1 and Mkk1 in the nematode-trapping (NT) fungus Arthrobotrys oligospora by multiple phenotypic comparison, and the regulation of conidiation and cell wall synthesis was analyzed using real-time PCR (RT-PCR). Both $\triangle A o B c k 1$ and $\triangle$ AoMkk1 mutants showed severe defects in vegetative growth, cell nucleus number, and stress resistance. Both the mutants were unable to produce spores, and the transcription of several genes associated with sporulation and cell wall biosynthesis was markedly downregulated during the conidiation stage. Further, cell walls of the $\triangle$ AoBck1 and $\triangle A O M k k 1$ mutants were severely damaged, and the Woronin body failed to respond to cellular damage. In particular, the mutants lost the ability to produce mycelial traps for nematode predation. Taken together, AoBck1 and AoMkk1 play a conserved role in mycelial growth and development, $\mathrm{CWI}$, conidiation, multi-stress tolerance, trap formation, and pathogenicity. We highlighted the role of AoBck1 and AoMkk1 in regulating the Woronin body response to cellular damage and cell nucleus development in A. oligospora.

Keywords: Arthrobotrys oligospora, cell wall integrity pathway, mitogen-activated protein kinases Bck1 and Mkk1, cell wall damage, Woronin body, trap formation

\section{INTRODUCTION}

The fungal cell wall is essential to maintain cellular structure and protect the cells from environmental stresses; its integrity is very important for fungal survival and adaption under adverse condition (Levdansky et al., 2010). The cell wall integrity (CWI) pathway is an important and conserved mitogen-activated protein kinase (MAPK) signaling pathway commonly found 
in fungi, which senses the cell wall stress signals produced by fungal cells during normal growth or environmental changes and responds in time (Turrà et al., 2014). The CWI pathway is one of the main regulatory pathways for fungal cell wall synthesis and integrity maintenance and is also related to the pathogenicity of pathogens (Hohmann, 2002). In yeast, the CWI pathway controls cell wall synthesis and cell cycle progression through a conserved MAP kinase cascade (Bck1, Mkk1/2, and Slt2) in response to cell surface stress; its signal transmission depends on the cascade phosphorylation reaction, where protein kinase $\mathrm{C}$ first activates the phosphorylation of Bck1, and Bck1 activates the phosphorylation of MKK1/2 which finally triggers the phosphorylation of Slt2, thereby transmitting signals to downstream effectors (Levin, 2005, 2011; Jin et al., 2015).

In recent years, the CWI pathway has also been characterized in several filamentous fungi, such as Aspergillus fumigatus (Valiante et al., 2009; Levdansky et al., 2010), Magnaporthe grisea (Zhao et al., 2005; Jeon et al., 2008), and Beauveria bassiana (Luo et al., 2012; Chen et al., 2014). These studies indicated that after the cascade components of the CWI pathway were destroyed in fungi, the mutant strains showed varying degrees of cell wall defects and increased their sensitivity to cell wall interfering agents, which affected their pathogenicity. In addition, the components of the CWI-MAPK cascade are also involved in a variety of biological processes, such as mycelial growth, conidiation, and environmental stress response. In Saccharomyces cerevisiae, Slt2 plays an important role in regulating the cell cycle and promoting cell polarization (Zarzov et al., 1996). Similarly, the homologous gene of Slt2 is necessary for conidiation in several filamentous fungi such as A. fumigatus (Valiante et al., 2009) and Metarhizium robertsii (Chen et al., 2016). Slt2 also plays a role in osmotic pressure regulation in several fungi, such as B. bassiana (Chen et al., 2014), M. robertsii (Chen et al., 2016), and Botrytis cinerea (Liu et al., 2011). Evidently, the CWI-MAPK signaling pathway cascade participates in the regulation of multiple biological processes in different pathogenic fungi.

Nematode-trapping (NT) fungi are an important natural enemy of nematodes. The vegetative hyphae of NT fungi can specifically form a variety of traps (such as adhesive networks, adhesive knobs, and constricting rings) to capture and infect nematodes (Su et al., 2017; Yang et al., 2020). The trapping initiates a series of processes, including adhesion, penetration, and immobilization of nematodes (Nordbring-Hertz, 2004; Yang et al., 2011). Trap formation is a prerequisite for NT fungi to capture nematodes and is also an important indicator for their lifestyle switch from a saprophytic to a predacious stage (Nordbring-Hertz et al., 2001; Su et al., 2017). Arthrobotrys oligospora is a species of NT fungi which usually forms adhesive networks (traps), and when prey, such as nematodes, appear in the environment, the vegetative hyphae of A. oligospora specialize to form adhesive networks for nematode predation (Nordbring-Hertz, 2004). In 2011, we sequenced the genome of A. oligospora, and proteomic analysis suggested that the $\mathrm{G}$ protein signaling pathway might be involved in trap formation and pathogenicity (Yang et al., 2011). Subsequently, Yang et al. (2020) proved that $G$ protein signaling plays an indispensible role in trap formation and pathogenicity of A. oligospora.
Genomic analysis showed that there is a conserved MAPK cascade pathway in A. oligospora that is homologous to Bck1Mkk1-Slt2 in yeast. Recently, we found that orthologous Slt2 plays an important role in two NT fungi, A. oligospora and Monacrosporium haptotylum (syn. Dactylellina haptotyla), which is involvement in mycelial growth and stress resistance. Specifically, the deletion of Slt2 abolished conidiation and trap formation (Zhen et al., 2018). Similarly, a Slt2 deletion mutant of A. oligospora displayed severe defects in vegetative growth, conidiation, and trap morphogenesis (Chen et al., 2021). However, the functions of Bck1 and Mkk1 upstream of Slt2 are poorly understood in A. oligospora and NT fungi specifically. In this study, orthologous A. oligospora Bck1 (AoBckl) and Mkk1 (AoMkk1) were characterized via multi-phenotypic analyses, and their regulatory mechanisms in conidiation and stress resistance were analyzed by real-time PCR (RT-PCR).

\section{MATERIALS AND METHODS}

\section{Fungal Strains, Plasmid Vector, and Culture Conditions}

Arthrobotrys oligospora (ATCC24927) and the mutant strains $\triangle A o B c k 1$ and $\triangle A o M k k 1$ were cultured on potato dextrose agar (PDA) plates at $28^{\circ} \mathrm{C}$. Saccharomyces cerevisiae strain FY834 (ATCC90845) was cultured in YPD (10 g/L yeast extract, $20 \mathrm{~g} / \mathrm{L}$ peptone, and $20 \mathrm{~g} / \mathrm{L}$ glucose) broth or solid YPDA medium (YPD with $16 \mathrm{~g} / \mathrm{L}$ agar). Plasmids pRS426 and pCSN44 were used to construct the recombinations, and they were maintained in the Escherichia coli strain DH5 $\alpha$ (TaKaRa, Shiga, Japan). PDAS medium (PDA supplemented with $0.6 \mathrm{M}$ sucrose) was used for protoplast regeneration, and cornmeal-molasses-yeast (CMY), tryptone-glucose (TG), and tryptone yeast-extract glucose agar (TYGA) media were used to analyze mycelial growth and related phenotypic traits, as previously described (Ma et al., 2020). Caenorhabditis elegans (strain N2) worms were incubated in an oatmeal medium at $26^{\circ} \mathrm{C}$ for the bioassays (Xie et al., 2020).

\section{Analysis of the AoBck1 and AoMkk1 Sequence}

The sequences of AoBck1 (AOL_s00054g475) and AoMkk1 (AOL_s00076g699) were retrieved based on the homologous sequences of the model fungi $S$. cerevisiae, A. nidulans, and $N$. crassa by performing BLASTP searches in the NCBI database. Homologous sequences of $A o B c k 1$ and $A o M k k 1$ in diverse fungi were searched using the BLAST algorithm in GenBank, and the DNAman software was used to align and examine their similarity. The Expasy-Compute $\mathrm{pI} / \mathrm{MW}$ tool $^{1}$ was used to calculate the theoretical isoelectric point $(p I)$ and molecular weight (MW) of AoBck1 and AoMkk1.

\section{Disruption of $A \circ B c k 1$ and $A \circ M k k 1$ Genes}

The AoBck1 and AoMkk1 genes were disrupted using homologous recombination, as previously described (Tunlid et al., 1992; Colot et al., 2006). Briefly, 5' and $3^{\prime}$ flanking sequences of the

${ }^{1}$ https://www.expasy.org/resources/compute-pI-mw 
target gene and the hygromycin-resistance gene cassette $(h p h)$ were amplified from A. oligospora and pCSN44 with paired primers (Supplementary Table $\mathbf{S 1}$ ), respectively. Then, three DNA fragments and linearized pRS426 (digested with EcoRI and $X h o I$ ) were co-transformed into $S$. cerevisiae using electroporation. Finally, the recombinant plasmids, pRS426AoBck1-hph and pRS426-AoMkk1-hph, were individually transformed into A. oligospora protoplasts, as described previously (Yang et al., 2018). Southern blots and PCR analyses were used to further verify the transformant colonies grown on the PDAS medium (Tunlid et al., 1999). The Plant Genomic DNA Kit (TaKaRa) was used to extract genomic DNA from fungal strains, and their DNA were individually digested with restriction enzymes SphI and NheI for Southern blot analysis. The North2South Chemiluminescence Hybridization and Detection Kit (Pierce, Rockford, IL, United States) was used for the Southern blots according to the manufacturer's instructions.

\section{Comparison of Mycelial Growth, Morphology, and Conidiation}

The wild-type (WT) A. oligospora strain and the mutant strains $\triangle A o B c k 1$ and $\triangle A o M k k 1$ (two independent transformants were used for phenotypic analysis) were cultured on PDA plates at $28^{\circ} \mathrm{C}$ for 6 days, following which $7 \mathrm{~mm}$ diameter hyphal discs of each strain were inoculated separately in PDA, TYGA, and TG media at $28^{\circ} \mathrm{C}$ for 3-7 days, and the mycelial growth rate and colony morphology were observed at specific time intervals (Xie et al., 2019). To assess the conidiation capacity of each strain, the fungal strains were cultured on CMY medium at $26^{\circ} \mathrm{C}$ for 15 days, and the conidial yield was determined as previously described (Liu et al., 2017). The hyphae of the WT and mutant strains were stained with $20 \mu \mathrm{g} / \mathrm{ml}$ calcium fluoride fluorescent white (CFW; SigmaAldrich, St. Louis, MO, United States) to observe the mycelial morphology. To observe the mycelial septum and cell nucleus, the hyphae of the WT and mutant strains were stained with $20 \mu \mathrm{g} / \mathrm{ml}$ 4',6-diamidino-2-phenylindole (DAPI; Sigma-Aldrich, St. Louis, MO, United States) for $30 \mathrm{~min}$, then washed three times with phosphate buffer ( $\mathrm{pH}$ 6.8-7.2), and further stained with $80 \mu \mathrm{g} / \mathrm{ml} \mathrm{CFW}$ for $5 \mathrm{~min}$. The samples were observed using an inverted fluorescence microscope (Carl Zeiss, Heidenheim, Germany). Mycelial morphology was observed via scanning electron microscopy (SEM) 7 days post PDA culture (Quanta-200; FEI, Hillsboro, OR, United States), and cell wall damage and the Woronin body of hyphae were further examined via transmission electron microscopy (TEM; Hitachi, Tokyo, Japan) $28 \mathrm{~h}$ post PD culture, respectively. Samples for the SEM and TEM were treated as previously described (Zhang et al., 2013, 2019b).

\section{Stress Assays}

Hyphal discs (7 mm diameter) of each strain were incubated on TG medium alone (control) or supplemented with chemical stressors like Congo red and SDS (cell wall interfering agents), $\mathrm{NaCl}$ and sorbitol (osmotic agents), $\mathrm{H}_{2} \mathrm{O}_{2}$ (oxidant), and menadione. The colonies were cultured at $28^{\circ} \mathrm{C}$ for 6 days, and the relative growth inhibition (RGI) rate of each strain was calculated on different media to evaluate the response to chemical stress, as previously described (Liu et al., 2017; Zhen et al., 2018). In addition, the fungal strains were incubated on PDA medium at $28^{\circ} \mathrm{C}$ for 2 days, then placed at different temperatures, such as $28,34,38,40,42$, and $44^{\circ} \mathrm{C}$ for $8 \mathrm{~h}$, and then left to grow at $28^{\circ} \mathrm{C}$ until the 6th day. The colony diameter was measured, and RGI values were calculated as described above. All the stress assays for each strain were repeated thrice.

\section{Trap Formation and Nematocidal Activity Analyses}

Hyphal discs (7 mm diameter) of each strain were incubated on water agar (WA, 20\%) medium at $28^{\circ} \mathrm{C}$ for 5 days. Then, approximately 300 wild-type L4-stage C. elegans nematodes were added to each WA plate to induce trap formation and nematode predation. A microscope (Olympus, Tokyo, Japan) was used to observe and count the traps and the captured nematodes from each plate at $48 \mathrm{~h}$. The bioassays for each strain were repeated three times.

\section{Transcriptional Analyses of Selected Genes}

The hyphae of the WT and mutant strains were cultured on TYGA at $28^{\circ} \mathrm{C}$, and the mycelia samples were collected on the 3rd, 5th, and 7th day, and the total RNA of mycelia samples was isolated using an AxyPrep multisource RNA miniprep kit (Axygen, Jiangsu, China), and then reverse-transcribed into cDNA using a FastQuant RT kit with gDNase (TaKaRa). The cDNA samples of each strain were used as templates for RT-PCR analysis with specific paired primers (Supplementary Table S2). The $\beta$-tubulin gene of $A$. oligospora was used as an internal standard, and the transcriptional level of the genes associated with conidiation and cell wall synthesis was calculated using the $2^{-\Delta \Delta \mathrm{Ct}}$ method (Livak and Schmittgen, 2001). Three replicates were performed in the RT-PCR experiments, and the RT-PCR analysis for each gene was repeated three times. The relative transcript level (RTL) of candidate genes was estimated as the transcript ratio of each mutant vs. the WT.

\section{Statistical Analyses}

GraphPad Prism version 8.0 (GraphPad Software, San Diego, CA, United States) was used for statistical analysis of data from three repeated experiments, and the variance between the WT and mutants was estimated by the Tukey's honest significant difference (HSD) test. Value of $p<0.05$ was considered significant.

\section{RESULTS}

\section{Sequence Analyses of $A \circ B c k 1$ and AoMkk1}

The nucleotide sequences of $A o B c k 1$ (8,518 bp with four introns) and AoMkk1 (4,525 bp with four introns) were retrieved from 
the fungus $A$. oligospora. AoBck1 encodes a polypeptide of 1,816 amino acids with a predicted MW of $198 \mathrm{kDa}$ and a pI of 8.31. AoMkk1 encodes a polypeptide of 478 amino acids with a predicted $\mathrm{MW}$ of $52 \mathrm{kDa}$ and $\mathrm{pI}$ of 9.09 . Both the encoded proteins contain multiple conserved domains and motifs, such as the protein kinase-like domain superfamily (IPR011009), protein kinase domain (IPR000719), ATP binding site of protein kinase (IPR017441), and active site of Ser/Thr protein kinase (IPR008271). It was found that both AoBck1 and AoMkk1 contain the conserved active site motif "-D[L/I/V] $\mathrm{K}-$," AoBck1 contains the conservative motif "-G[S/T][V/P] $[\mathrm{F} / \mathrm{M}][\mathrm{W} / \mathrm{Y}] \mathrm{M}[\mathrm{A} / \mathrm{S}] \mathrm{PE}-”$ (Supplementary Figure S1A), and AoMkk1 contains the conservative motif "-[S/T] $\mathrm{xxx}[\mathrm{S} / \mathrm{T}]$ in addition to "-D $[\mathrm{L} / \mathrm{I} / \mathrm{V}]$ K-" (Supplementary Figure S1B). Moreover, AoBck1 shares a high degree of similarity (79.4 and $85.5 \%$ ) with the orthologs of the NT fungi Drechslerella stenobrocha (Liu et al., 2014) and D. haptotyla (Meerupati et al., 2013), respectively. Similarly, AoMkk1 also shares a high identity (89.4 and 93.1\%) with the orthologs of NT fungi $D$. stenobrocha (Liu et al., 2014) and D. haptotyla (Meerupati et al., 2013), respectively. In contrast, 21.1-59.3\% identity was found between $A o M k k 1 / A o B c k 1$ and the orthologs of other filamentous fungi.

\section{Verification of the Positive Transformants of AoBck1 and AoMkk1}

Single-gene disruption mutants of $A o B c k 1$ and $A o M k k 1$ were constructed by homologous recombination. The positive transformants were verified by PCR amplification with primers YZBck1-F/R and YZMkk1-F/R (Supplementary Table S1; Supplementary Figures S2A,B), respectively. To avoid the presence of false positives due to multi-site insertion or incorrect insertion sites, further identification was performed using Southern blots. Only one specific hybridization band was identified in the $\Delta A o B c k 1$ and $\Delta A o M k k 1$ mutants, respectively, indicating that there was no non-specific recombination in the $\triangle A o B c k 1$ and $\triangle A o M k k 1$ strains (Supplementary Figures S2Aa-c,Ba-c). Finally, two transformants for AoBck1 and AoMkk1 were confirmed to contain the correct mutations. A single strain from each mutant was selected for subsequent studies because the independent mutant strains for each gene showed similar phenotypic properties.

\section{AoBck1 and AoMkk1 Regulate Mycelial Growth, Morphology, and Cell Nucleus Number}

Deletion of $A o B c k 1$ and $A o M k k 1$ caused a significant reduction in mycelial growth in the PDA, TG, and TYGA media (Figures 1A,B). In addition, the WT strain had very dense aerial hyphae in the TYGA medium, whereas colonies of the $\triangle A o B c k 1$ and $\triangle A o M k k 1$ mutants were irregular and lacked aerial hyphae (Figure 1A). In addition, the mycelial tip of the third branch in the $\Delta A o B c k 1$ and $\Delta A o M k k 1$ mutants was longer than that of the WT strain (Figures 1C,D). After CFW staining, it was found that the hyphal widths of the $\triangle A o B c k 1$ and $\triangle A o M k k 1$ mutant strains were significantly smaller, curly short branches were increased, and the hyphal cell length was significantly uneven compared to the WT strain (Figures 2A-C). Moreover, the nuclei of the WT and the $\triangle A o B c k 1$ and $\triangle A o M k k 1$ mutant strains were observed by DAPI staining, and the number of nuclei in the mycelial cells of the $\Delta A o B c k 1$ and $\Delta A o M k k 1$ mutant strains was significantly reduced (only 2-4 nuclei per cell), whereas the hyphal cells of the WT strain contained 6-22 nuclei (Figures 2D,E).
A

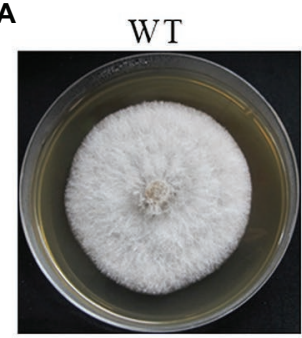

c WT $\triangle A o B c k 1$

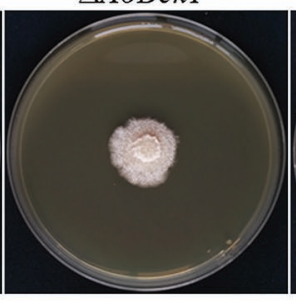

$\triangle A o B c k 1$

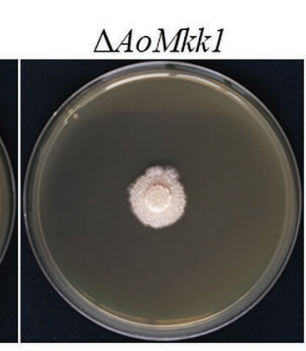

$\triangle A o M k k 1$
B

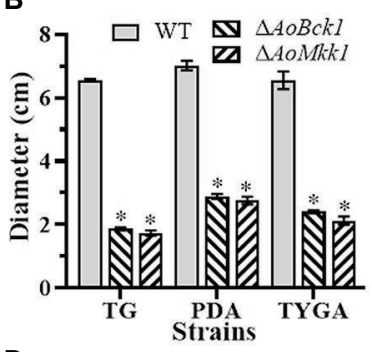

D

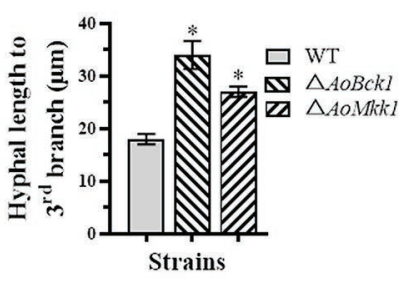

FIGURE 1 | Comparison of colonial and hyphal morphologies between the wild-type (WT) and mutants. (A) Colony morphology of the WT and mutants incubated on tryptone yeast-extract glucose agar (TYGA) medium for 5 days at $28^{\circ} \mathrm{C}$. (B) Colony diameters of the WT and mutants incubated on potato dextrose agar (PDA), TYGA, and tryptone-glucose (TG) media for 7 days. Error bars: SD from three replicates; asterisk: significant differences between mutant and WT ( $p<0.05$ ). (C) Mycelial morphologies of the WT and two mutants as observed by light microscopy. Arrows: mycelial tip and the third branch of mycelium. Bar $=20 \mu \mathrm{m}$. (D) The distance from the tip of the mycelium of each strain to the third branch. 
A

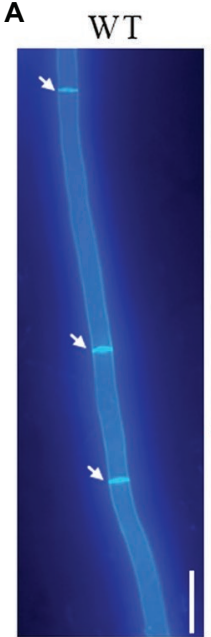

B

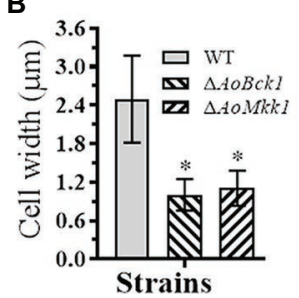

$\triangle A o B c k I$

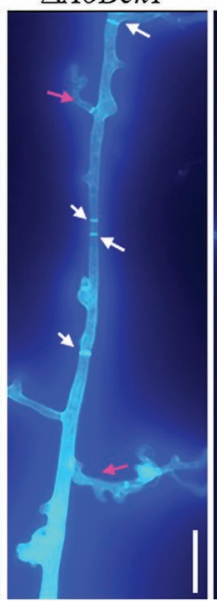

C

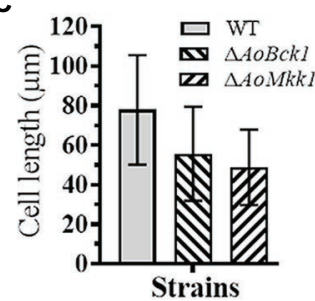

$\triangle A o M k k 1$

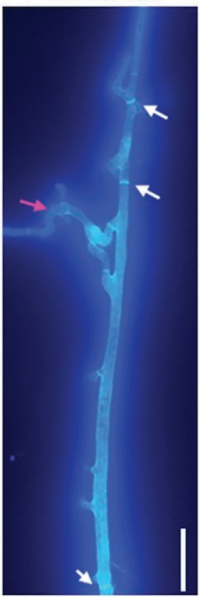

Strain
D WT $\triangle A o B c k 1 \triangle A o M k k 1$
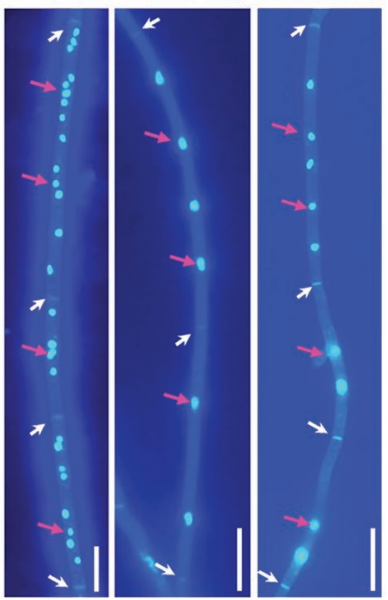

E

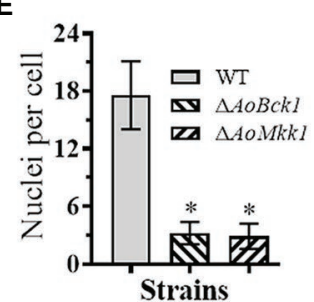

FIGURE 2 | Comparison of hyphal septum, branch, and cell nucleus between the WT and mutants. (A) Hyphal septa of the WT and mutants were stained with $20 \mu \mathrm{g} / \mathrm{ml}$ calcofluor white (CFW) after the fungal strains were incubated on cornmeal-molasses-yeast (CMY) medium for 7 days. White arrows: hyphal septa; red arrows: hyphae branch. Bar = $10 \mu \mathrm{m}$. (B) Comparison of mycelial width of each strain. (C) Comparison of mycelial cell length of the WT and mutants; 61 mycelial cells were randomly selected, and the width and length of hyphal cell were measured using ImageJ software. Error bars: SD from 61 replicates. (D) Hyphae of the WT and each mutant strain were stained with CFW and 4',6-diamidino-2-phenylindole (DAPI) after the fungal strains were grown for 7 days on CMY medium; samples were examined using an inverted fluorescence microscope. White arrows: septa; red arrows: cell nucleus. Bar $=10 \mu \mathrm{m}$. (E) Comparison of mycelial cell nuclei between the WT and each mutant strain. Thirty hyphal cells were randomly selected for counting cell nuclei. Error bars: SD from 30 replicates; an asterisk indicates a significant difference compared to the WT $(p<0.05)$.

\section{AoBck1 and AoMkk1 Are Necessary for Conidiation}

Deletion of $A o B c k 1$ and $A o M k k 1$ resulted in a deleterious effect in conidiation, and two mutants were unable to produce conidia (Figures 3A,B). In order to probe into the regulation of $A o B c k 1$ and $A o M k k 1$ for conidiation in A. oligospora, nine sporulation-related genes, $V e A, F l b C, N s d D, F l u G, \operatorname{Rod} A, \operatorname{Vos} A$, $V e l B, A s p B$, and $A b a A$, were selected, and their transcription was analyzed at different growth stages using RT-PCR. The fungus $A$. oligospora usually begins to form conidiophores for conidiation on the $3 \mathrm{rd}$ day, the number of conidiophores and conidia increase rapidly on the 5th day, and most conidiophores and conidia are produced on the 7th day. The expression levels of all genes were significantly downregulated in the $\triangle A o B c k 1$ mutant (Figure $3 \mathrm{C}$ ). Similarly, all genes involved in sporulation, except $N s d D$ and $A s p B$ at days 3, were significantly downregulated in the $\triangle A o M k k 1$ mutant (Figure 3D). Specifically, VelB and FluG were downregulated more significantly at day 3 and day 7 , respectively, in the $\triangle A o B c k 1$ mutant (Figure 3C). Our analysis indicated that $A o B c k 1$ and $A o M k k 1$ play important roles in the conidiation of A. oligospora.

\section{AoBck1 and AoMkk1 Play a Crucial Role in CWI}

Scanning electron microscopy observations showed that the mycelial surface of the $\Delta A o B c k 1$ and $\Delta A o M k k 1$ mutants was severely damaged, and the mycelia was fragmented and meshed (Figure 4A). Further TEM observation also revealed a similar phenomenon; the cell walls of the $\triangle A o B c k 1$ and $\triangle A o M k k 1$ mutants were loosely distributed outside the cell membrane; and their cell membranes were incomplete. In contrast, compact cell walls and distinctly outlined membranes were observed in the WT hyphae cells (Figure 4B). To further detect cell wall damage, the WT and the mutant strains $\triangle A o B c k 1$ and $\Delta A o M k k 1$ were cultivated in PD broth for $24 \mathrm{~h}$ and the mycelia of each strain were treated with cell wall-degrading enzymes, snailase, and cellulase. As a result, the hyphal cells of the $\triangle A o B c k 1$ and $\triangle A o M k k 1$ mutants released 3.5-3.7 and 6.04-6.12fold more protoplasts than that of the WT strain after 3 and $6 \mathrm{~h}$ treatments, respectively (Supplementary Figure S3). Moreover, three to four independent Woronin bodies were observed near the hyphal septa of the WT strain, and the injured hyphal septa were sealed by Woronin bodies (Figure 4C). The Woronin bodies are reduced in the mutant strains, although 
A

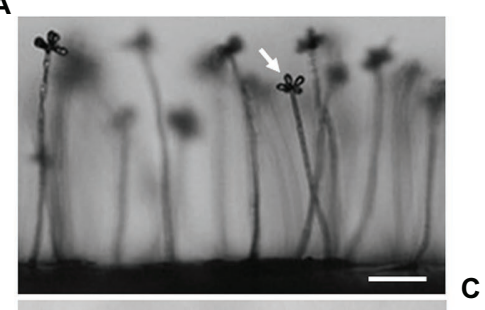

B

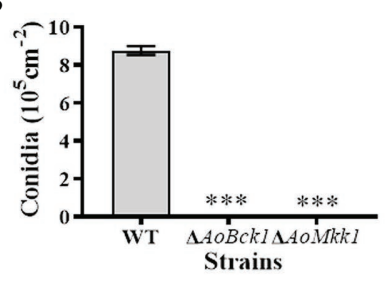

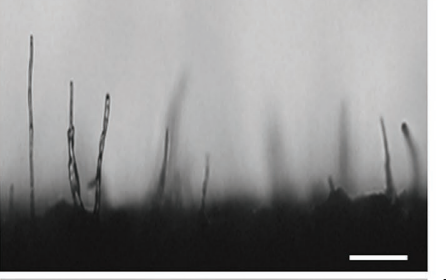

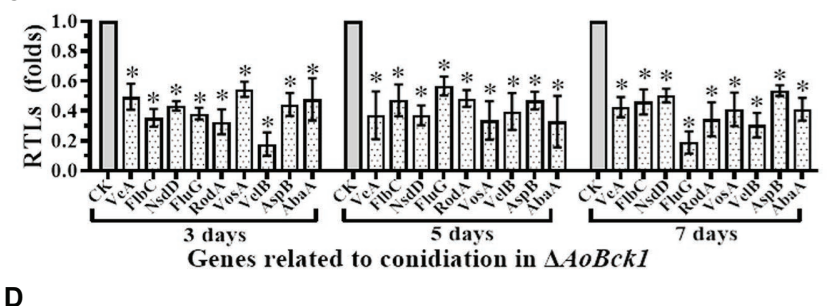

.

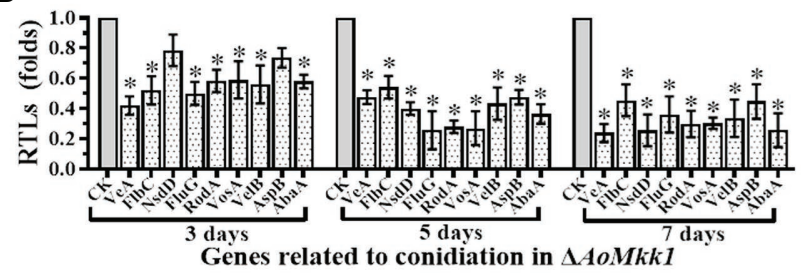

FIGURE 3 | Comparison of conidiation and transcript of sporulation-related genes between the WT and mutants. (A) Comparison of conidiophore and sporulation the WT and mutants on CMY medium. White arrows: conidium; Bar $=10 \mu \mathrm{m}$. (B) Conidial yields in the WT vs. $\Delta$ AoBck1 and $\Delta$ AoMkk1 mutants. Three asterisks: very significant difference between mutant and the WT $(p<0.001)$. (C) Relative transcript levels (RTLs) of selected genes involved in conidiation of the WT and $\triangle$ AoBck1 mutants at different time points. (D) RTLs of selected genes involved in conidiation of the WT and $\Delta$ AoMkk1 mutants at different time points. An asterisk indicates a significant difference between the mutants and the WT strain $(p<0.05)$. CK under given conditions, using CK with an RTL of 1 as the standard, statistical analysis was performed on the RTL of each gene in each mutant and the WT strain. An asterisk indicates a significant difference compared to the WT $(p<0.05)$.

they are near the opening of the septa (Figure 4C). Six genes of A. oligospora involved in cell wall biosynthesis were transcriptionally compared between the WT and mutant strains by RT-PCR, and it was observed that all the genes were downregulated in the $\Delta A o B c k 1$ and $\triangle A o M k k 1$ mutants compared to the WT strain (Figures $4 \mathrm{D}, \mathrm{E}$ ).

\section{AoBck1 and AoMkk1 Regulate Multiple Stress Responses}

Compared to the WT, the $\triangle A o B c k 1$ and $\triangle A o M k k 1$ mutants showed increased sensitivity to several chemical stressors like two cell wall interfering agents (Congo red and SDS) and two osmotic agents ( $\mathrm{NaCl}$ and sorbitol), whereas they were unaffected by oxidants $\mathrm{H}_{2} \mathrm{O}_{2}$ and menadione (Figure 5A). The RGI values of the $\Delta A o B c k 1$ mutant exposed to $0.2 \mathrm{M} \mathrm{NaCl}, 0.5 \mathrm{M}$ sorbitol, $0.07 \mathrm{mM}$ Congo red, and $0.7 \mathrm{mM}$ SDS were increased by $15.27,30.60,28.5$, and $27.1 \%$, respectively, as compared to the WT strain. At similar exposure concentrations of the chemical stressors as the $\Delta A o B c k 1$ mutant, the RGI values of the $\Delta A o M k k 1$ mutant colonies with sorbitol, $\mathrm{NaCl}$, Congo red, and SDS were increased by $16.02,20.32,33.5$, and $27.4 \%$, respectively, in comparison to the WT (Figure 5B). Moreover, the heat shock results showed that the colony growth of the $\triangle A o B c k 1$ and $\Delta A o M k k 1$ mutants was significantly inhibited at $42^{\circ} \mathrm{C}$ compared to the WT (Figure 5C; Supplementary Figure S4).

\section{AoBck1 and AoMkk1 Are Required for Trap Formation and Pathogenicity}

The WT strain began to produce immature traps containing 1-2 hyphal loops $12 \mathrm{~h}$ post nematode induction, and mature traps composed of multiple hyphal loops began to form at $24 \mathrm{~h}$. Almost, all the added nematodes were captured by the WT strain at $36 \mathrm{~h}$ and were digested at $48 \mathrm{~h}$. The WT strain produced approximately 25 traps $\mathrm{cm}^{-2}$ at $48 \mathrm{~h}$, whereas the $\triangle A o B c k 1$ and $\triangle A o M k k 1$ mutants did not produce any traps (Figures 6A,B). Interestingly, approximately $90.1 \%$ of the nematodes were captured by the WT strain at $48 \mathrm{~h}$, whereas no nematodes were captured by the $\Delta A o B c k 1$ and $\triangle A o M k k 1$ mutants (Figure 6C).

\section{DISCUSSION}

Nematodes and fungi depend on and communicate through elaborate networks of signaling. The MAPK signaling cascades are key evolutionarily conserved signal transducers in all eukaryotes, and several MAPKs have been identified in A. oligospora, including Slt2 (Zhen et al., 2018; Chen et al., 2021), Fus3 (Chen et al., 2021), Hog1 (Kuo et al., 2020), and Ime2 (Xie et al., 2020). In filamentous ascomycetes, the Slt2MAPK cascade pathway is an important signaling pathway, 
A
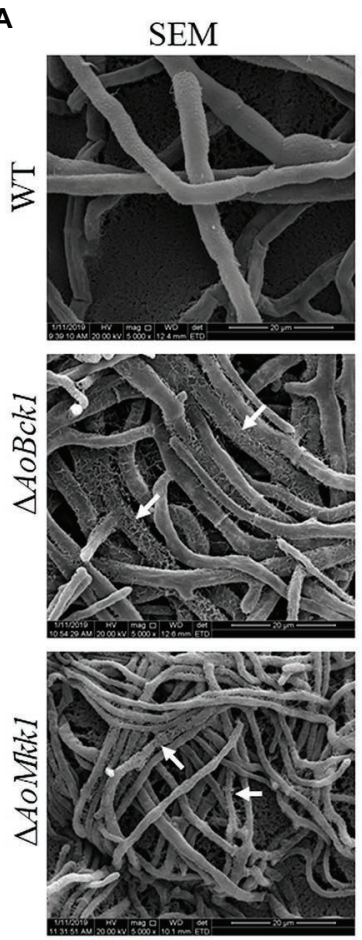
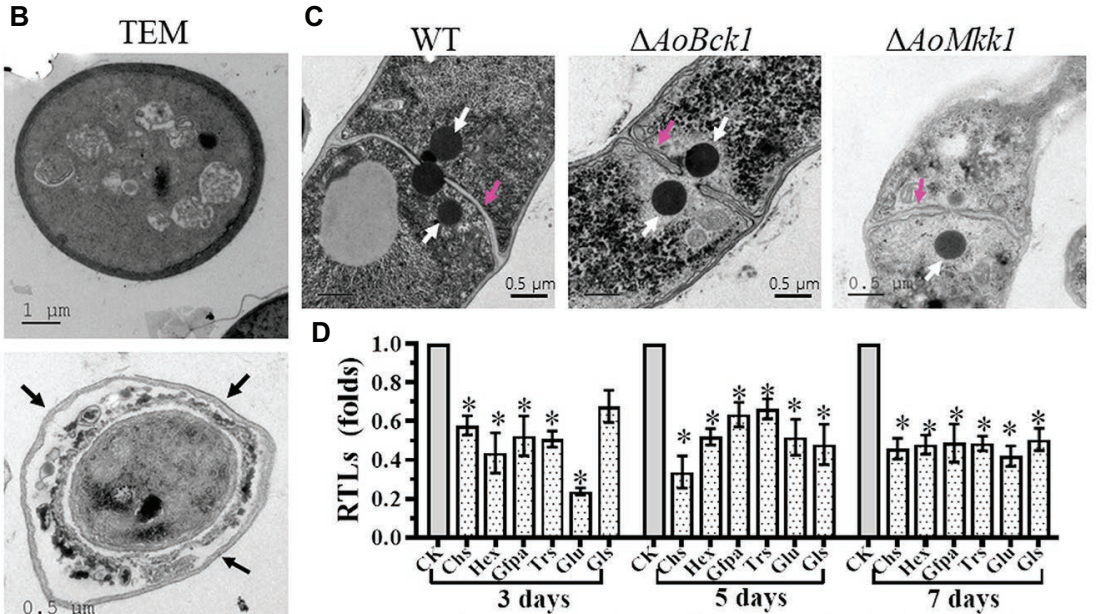

E
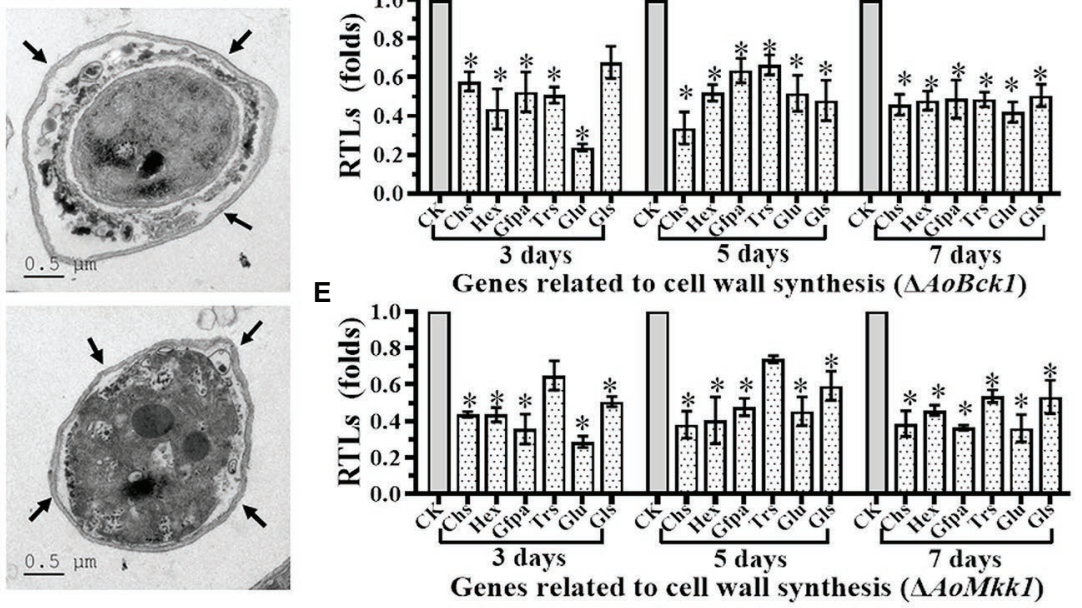

FIGURE 4 | Observation of mycelial morphology using scanning electron microscopy (SEM) and transmission electron microscope (TEM), and transcript of genes associated with cell wall biosynthesis in the WT and mutants. (A) Mycelia of each strain were examined using SEM. Bar $=20 \mu \mathrm{m}$. White arrows: the fragmented mycelial surface; (B) Observation of hyphal cells by TEM. Bar $=1 \mu \mathrm{m}$ (WT) or $0.5 \mu \mathrm{m}$ (mutants). Black arrow: separation of cell wall and cell membrane.

(C) Comparison of Woronin bodies between the WT and mutants. The hyphae of each strain cultured at $28^{\circ} \mathrm{C}$ in PD broth for $18 \mathrm{~h}$, after which, post fixing in $2.5 \%$ glutaraldehyde, samples were examined by TEM. Red arrow: hyphal septum; white arrows: Woronin body. Bar $=0.5 \mu \mathrm{m}$. (D) RTLs of selected genes involved in cell wall synthesis of the WT and $\Delta$ AoBck1 mutant at different time points. (E) RTLs of selected genes involved in cell wall synthesis of the WT and $\Delta$ AoMkk1 mutant at different time points. CK under given conditions, using CK with an RTL of 1 as the standard, statistical analysis was performed on the RTL of each gene in each mutant and the WT strain. An asterisk indicates a significant difference compared to the WT $(p<0.05)$.

which consists of $B c k 1, M k k 1 / 2$, and Slt2 in S. cerevisiae (Levin, 2005). In this study, we characterized the orthologs of Bck1 and Mkk1 (AoBck1 and AoMkk1) in the NT fungus A. oligospora. It was found that deletion of AoBck1 or AoMkk1 affects diverse phenotypic traits in A. oligospora.

In several filamentous fungi, deletion of $B c k 1$ and $M k k 1 / 2$ caused defects in mycelial growth and development. For example, the $\Delta B c k 1, \Delta M k k 2$, and $\Delta M p k a$ mutants showed significantly reduced hyphal growth, elevated branching, and abnormal hyphal structure in A. fumigatus (Valiante et al., 2009). In Magnaporthe oryzae, the $\Delta M p s 1$ mutant had severe defects in aerial hyphal growth (Xu et al., 1998). In A. oligospora, deletion of AoSlt2 resulted in a serious defect in mycelial growth and aerial hyphae development in A. oligospora (Zhen et al., 2018; Chen et al., 2021). Similar to the $\Delta$ AoSlt2 mutant, the $\Delta A o B c k 1$ and $\Delta A o M k k 1$ mutants also showed a significant reduction in mycelial growth and aerial hyphae, where the colonies of the $\Delta A o B c k 1$ and $\Delta$ AoMkk1 mutants became irregular and hyphal branches were increased compared to the WT strain. Importantly, deletion of $A o B c k 1$ and $A o M k k 1$ resulted in a significant reduction in the cell nucleus number. Thus, AoBck1 and $A o M k k 1$ play a conserved role in the regulation of mycelial growth and aerial hyphae in diverse fungi, and they also regulate cell nucleus number in A. oligospora.

Arthrobotrys oligospora is a typical NT fungal species that can reproduce asexually by producing abundant conidia (Zhang et al., 2019b). In this study, the $\Delta A o B c k 1$ and $\Delta A o M k k 1$ mutants completely lost their ability for conidiation, which is similar to the $\Delta$ AoSlt2 mutant (Zhen et al., 2018; Chen et al., 2021). Similar defects in conidiation were also found in other fungi, such as the significantly reduced conidial yields of the Slt2MAPK cascade mutants in $M$. robertsii. Moreover, deletion of $B c k 1, M k k 1$, and Slt2 caused a decrease in the number of conidia in M. oryzae (Xu et al., 1998; Zhao et al., 2005; Jeon et al., 2008). Regarding the defect in conidiation, the transcription of genes associated with conidiation was substantially downregulated in the $\triangle A o B c k 1$ and $\triangle A o M k k 1$ mutants compared to the WT strain. $A b a A$ is a central developmental regulator in the conidiation of $A$. nidulans and other filamentous fungi (Park and Yu, 2012; Krijgsheld et al., 2013). Deletion of AbaA abolished aerial conidiation and submerged blastospore formation in vitro despite no negative impact on hyphal growth in various media in B. bassiana (Zhang et al., 2019a). Moreover, we found that the orthologous gene of $\mathrm{VelB}(\mathrm{AoVelB})$ is essential for conidiation in $A$. oligospora, as the $\triangle A o V e l B$ mutant is unable 


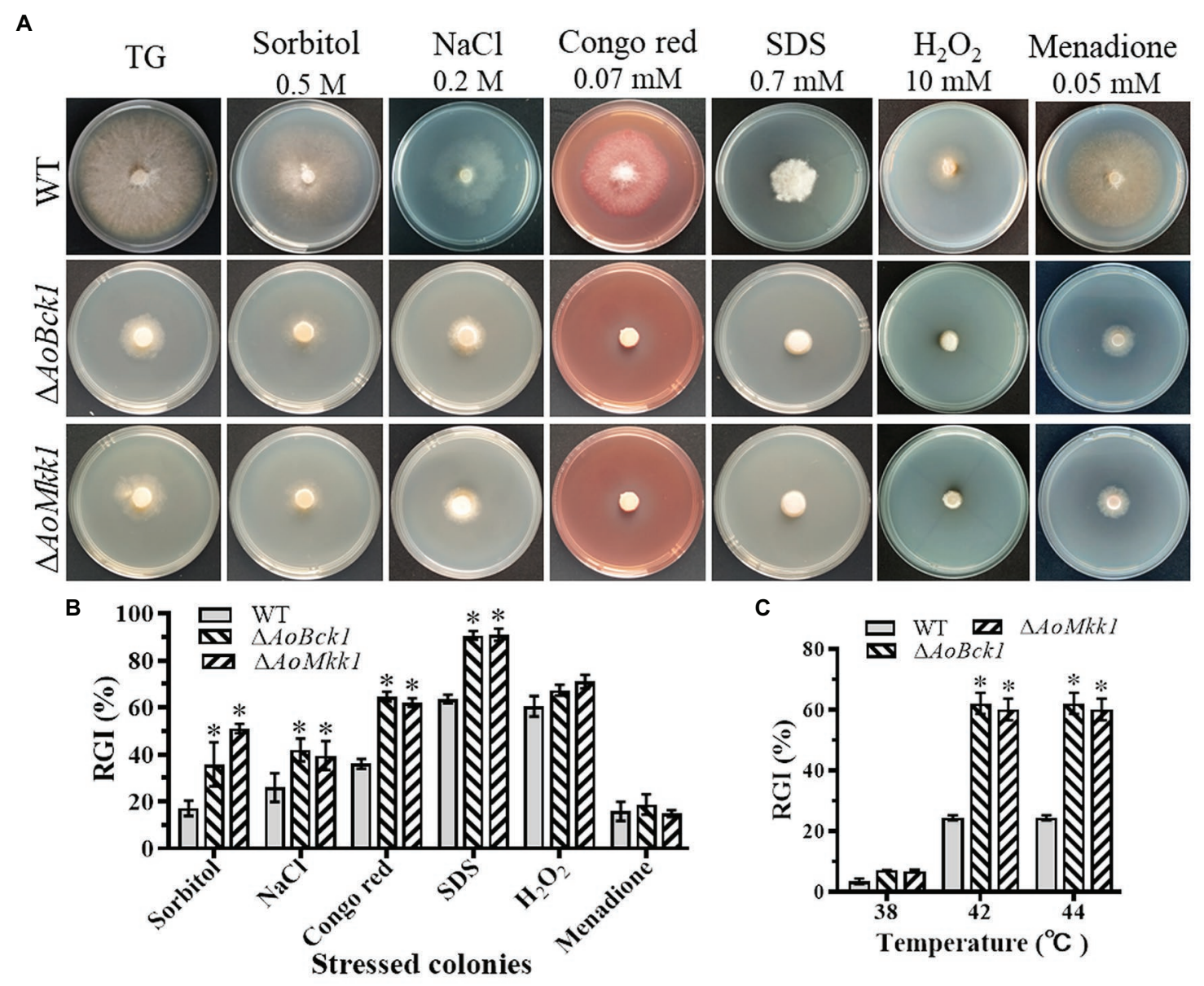

FIGURE 5 | Comparison of stress response between the WT and mutants. (A) Colonial morphology of fungal strains under stresses of osmotic, oxidative, and cell wall interfering agents stress. (B) Relative growth inhibition (RGI) of fungal colonies after 7 days grown at $28^{\circ} \mathrm{C}$ on $\mathrm{TG}$ plates supplemented with $0.2 \mathrm{M} \mathrm{NaCl,} 0.5 \mathrm{M}$ sorbitol, $0.07 \mathrm{mM}$ Congo red, $0.7 \mathrm{mM} \mathrm{SDS}, 10 \mathrm{mM} \mathrm{H}_{2} \mathrm{O}_{2}$, and $0.05 \mathrm{mM}$ menadione. (C) RGI values of the WT and each mutant grown at different temperatures. An asterisk indicates a significant difference compared to the WT $(p<0.05)$.

to produce spores (Zhang et al., 2019b). These results indicate that Bck1, Mkk1, and Slt2 play a conserved role in conidiation in A. oligospora and other fungi.

In recent years, an increasing number of studies have suggested that $B c k 1, M k k 1 / 2$, and Slt2 play a crucial role in fungal cell wall synthesis and integrity maintenance (Xu et al., 1998; Hohmann, 2002; Chen et al., 2014). In this study, deletion of $A o B c k 1$ and AoMkk1 caused severe damage to the cell wall of $A$. oligospora. Interestingly, protoplasts released by the $\Delta A o B c k 1$ and $\triangle A o M k k 1$ mutants were significantly increased after treatment with cell wall-lysing enzymes. Similarly, the $\Delta B c k 1$, $\Delta M k k 1$, and $\Delta$ Slt2 mutants released more protoplasts after treatment with cell wall-lysing enzymes, and their cell walls become thinner or more electron-transparent in B. bassiana (Chen et al., 2014). In M. oryzae, Mps1, Bck1, and Mkk1 were necessary to maintain CWI, and their mutants were highly sensitive to cell wall-degrading enzymes (Xu et al., 1998; Zhao et al., 2005; Jeon et al., 2008). Interestingly, the transcripts of several genes related to cell wall biosynthesis were significantly downregulated in the $\triangle A o B c k 1$ and $\triangle A o M k k 1$ mutants, which was consistent with the phenotypic alterations. In addition, the Woronin body is a peroxisome-derived dense-core vesicle that is unique to several genera of filamentous ascomycetes (Momany et al., 2002), which is involved in sealing septal pores in response to cellular damage (Navarro-Espíndola et al., 2020). The Woronin body of A. oligospora also plays an important regulatory role in conidiation, trap formation, stress resistance, and adaptation to nutrient-deficient environments (Liang et al., 2017). In this study, although Woronin bodies seemed to be present in the mutants, they were unable to seal the damaged septal pores and failed to respond to cellular damage. These results indicate that the Slt2-MAPK cascade pathway plays a conserved role in regulating CWI. Furthermore, AoBck1 and $A o M k k 1$ are involved in regulating the normal response of the Woronin body to cell damage.

It is important to adapt to altered environments for the vegetative growth, development, and reproduction in yeast and filamentous fungi. The $\triangle A o B c k 1$ and $\triangle A o M k k 1$ mutants were more sensitive to some chemical stressors than the WT strain but were unaffected by the oxidants used in this study. 


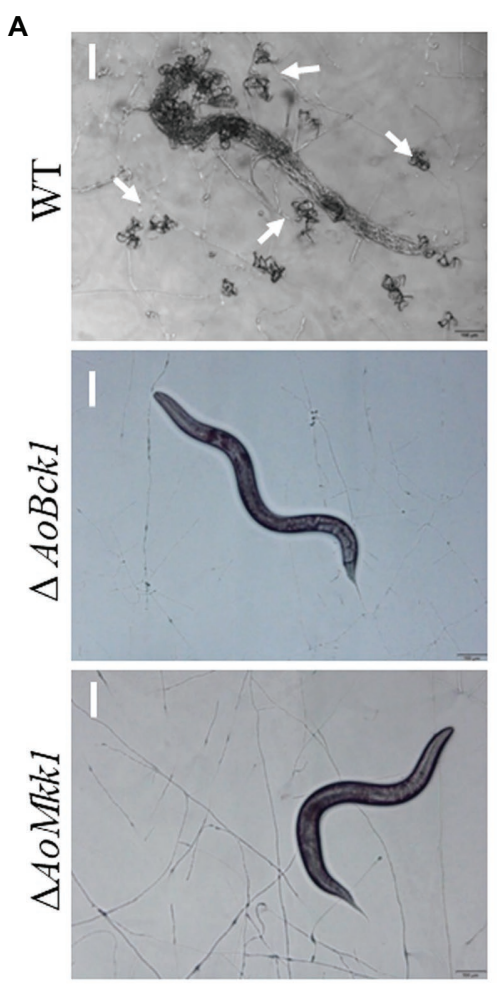

B

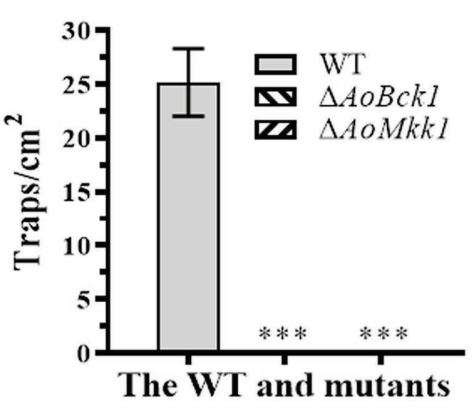

C

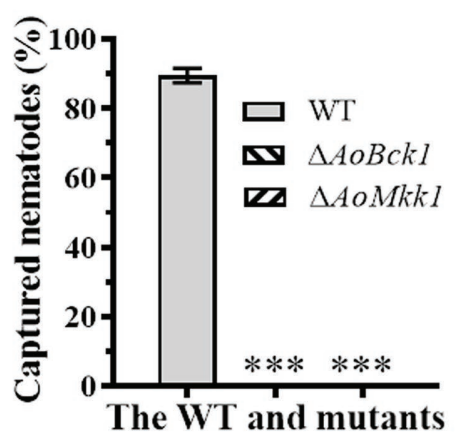

FIGURE 6 | Comparison of trap formation and nematocidal activity between the WT and mutants. (A) Trap formation of fungal strains induced by nematodes at 48 h. White arrows: Traps. Bar: $50 \mu \mathrm{m}$. (B) Comparison of traps produced by the WT and mutants at 48 h. (C) Comparison of captured nematodes by the WT and mutant strains at $48 \mathrm{~h}$. The asterisk indicates a significant difference compared to the WT $(p<0.05)$.

Similar to our observation, the $\Delta B c k 1, \Delta M k k 1$, and $\Delta$ Slt2 mutants of B. bassiana showed high cell sensitivity to $\mathrm{NaCl}$ and sorbitol, but negligible responses to oxidative stress by menadione or $\mathrm{H}_{2} \mathrm{O}_{2}$ were observed (Chen et al., 2014). Our previous study found that the sensitivity of the $\Delta$ AoSlt 2 and $\Delta M h$ Slt2 mutant strains to osmotic agents increased, but their sensitivity to oxidants also increased (Zhen et al., 2018). In addition, the $\triangle A o B c k 1$ and $\triangle A o M K K 1$ mutants were more sensitive to heat shock. Similarly, deletion of Slt2 in B. bassiana also resulted in increased cell sensitivity to high temperatures (Luo et al., 2012). These results show that AoBck1 and AoMkk1 regulate multi-stress tolerance in A. oligospora and other fungi.

The traps are an important feature for A. oligospora and other NT fungi to capture nematodes and are essential for their survival and virulence (Nordbring-Hertz, 2004). Deletion of $A o B c k 1$ and $A o M k k 1$ in A. oligospora resulted in the complete loss of the ability to form traps and the inability to capture nematodes, which is consistent with a previous report regarding AoSlt2 (Zhen et al., 2018; Chen et al., 2021). Similar results were also found in other fungi, such as the virulence of the $\Delta B c k 1, \Delta M k k 1$, and $\Delta$ Slt2 mutant strains being significantly reduced in B. bassiana (Luo et al., 2012; Chen et al., 2014) and $M$. robertsii (Chen et al., 2016). In M. oryzae, the $\Delta B c k 1$ mutant was nonpathogenic to susceptible rice seedlings (Jeon et al., 2008), the $\Delta M k k 1$ mutant lost the ability to infect the host (Zhao et al., 2005), and the $\Delta M p s 1$ mutant was defective in appressorium penetration (Xu et al., 1998). Moreover, deletion of one or two components of the CWI-regulated MAP kinase also impaired the virulence (Mehrabi et al., 2006; Jiang et al., 2018). These results show that CWI-regulated MAP kinases play a crucial role in the pathogenicity of diverse pathogens.

In this study, we found that AoBck1 and AoMkk1 not only participate in the regulation of several important functions, such as CWI, conidiation, multi-stress tolerance, and pathogenicity, but also regulate additional biological processes in A. oligospora, including the Woronin body response to cellular damage and cell nucleus development. Accordingly, we illustrated a schematic model for the regulation of $A o B c k 1$ and $A o M k k 1$ in A. oligospora (Figure 7). Arthrobotrys oligospora perceives nematode or cell wall stress signals through receptors at the surfaces of the cell membrane (Rsm), which are transferred into the intracellular environment and interact with the guanine nucleotide exchange factor Rom2 to activate the small GTPase Rho1, followed by activation of the protein kinase Pkc ( $\mathrm{Su}$ et al., 2017; Heinisch and Rodicio, 2018), which triggers the CWI-MAPK cascade and drives the transcription of downstream genes associated with multiple phenotypic traits. However, the role of Rsm, Rho1, and Pkc should be further verified. Our results lay a good foundation for revealing the mechanisms 


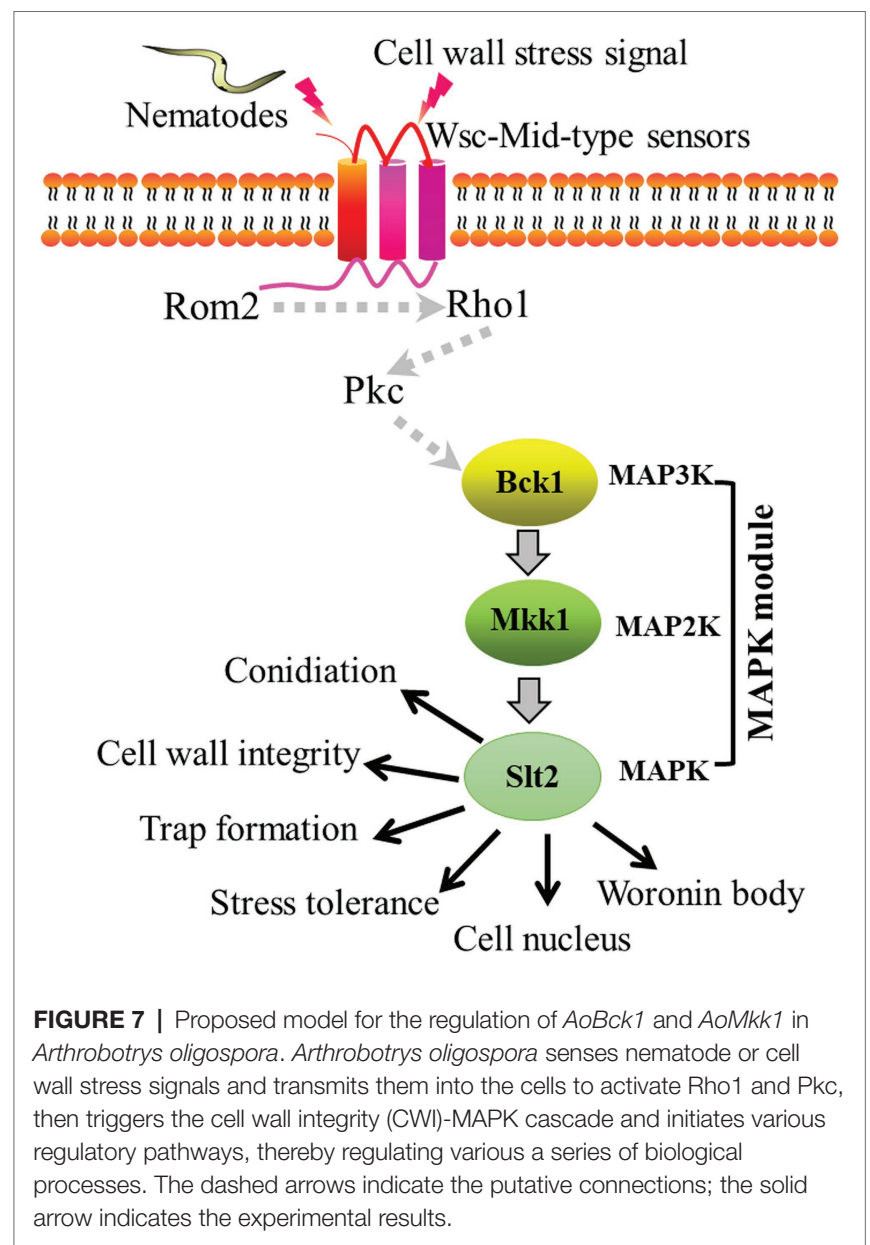

of trap formation and lifestyle switching in A. oligospora and other NT fungi.

\section{CONCLUSION}

We characterized orthologs of S. cerevisiae Bck1 and Mkk1 in the NT fungus A. oligospora. Both AoBck1 and AoMkk1 play an important role in vegetative growth, CWI, and stress resistance. In particular, $A o B c k 1$ and $A o M k k 1$ are required for conidiation and trap formation, and they also play roles in regulation of cell nucleus development and Woronin body response to cell damage in A. oligospora. Our findings provide a basis for investigating the role and mechanism of MAPK in vegetative

\section{REFERENCES}

Chen, S. A., Lin, H. C., Schroeder, F. C., and Hsueh, Y. P. (2021). Prey sensing and response in a nematode-trapping fungus is governed by the MAPK pheromone response pathway. Genetics 217:iyaa008. doi: 10.1093/genetics/ iyaa008

Chen, X., Xu, C., Qian, Y., Liu, R., Zhang, Q., Zeng, G., et al. (2016). MAPK cascade-mediated regulation of pathogenicity, conidiation and tolerance to abiotic stresses in the entomopathogenic fungus Metarhizium robertsii. Environ. Microbiol. 18, 1048-1062. doi: 10.1111/1462-2920.13198 growth, CWI, cell nucleus development, and pathogenicity of NT fungi.

\section{DATA AVAILABILITY STATEMENT}

The original contributions presented in the study are included in the article/Supplementary Material, further inquiries can be directed to the corresponding author.

\section{BIOSECURITY STATEMENT}

The strains and other materials used in this study are not applicable for biosecurity.

\section{AUTHOR CONTRIBUTIONS}

JinY conceived and designed the study. MX and JinY wrote the manuscript. MX conducted the experiments. JiaY, KJ, NB, $\mathrm{MZ}$, and YZ analyzed the data. JinY, K-QZ, and MX revised the manuscript. All authors contributed to the article and approved the submitted version.

\section{FUNDING}

This research described here was supported by the National Natural Science Foundation of China (nos. 31960556 and U1402265), the Applied Basic Research Foundation of Yunnan Province (202001BB050004), and the Yunnan Local Colleges Applied Basic Research Project (2017FH001-030).

\section{ACKNOWLEDGMENTS}

We would be grateful to Guo Yingqi (Kunming Institute of Zoology, Chinese Academy of Sciences) for her help of taking and analyzing the EM images.

\section{SUPPLEMENTARY MATERIAL}

The Supplementary Material for this article can be found online at: https://www.frontiersin.org/articles/10.3389/fmicb.2021.649582/ full\#supplementary-material

Chen, Y., Zhu, J., Ying, S. H., and Feng, M. G. (2014). Three mitogen-activated protein kinases required for cell wall integrity contribute greatly to biocontrol potential of a fungal entomopathogen. PLoS One 9:e87948. doi: 10.1371/ journal.pone.0087948

Colot, H. V., Park, G., Turner, G. E., Ringelberg, C., Crew, C. M., Litvinkova, L., et al. (2006). A high-throughput gene knockout procedure for Neurospora reveals functions for multiple transcription factors. Proc. Natl. Acad. Sci. U. S. A. 103, 10352-10357. doi: 10.1073/pnas.0601456103

Heinisch, J. J., and Rodicio, R. (2018). Protein kinase C in fungi-more than just cell wall integrity. FEMS Microbiol. Rev. 42, 22-39. doi: 10.1093/femsre/fux051 
Hohmann, S. (2002). Osmotic stress signaling and osmoadaptation in yeasts. Microbiol. Mol. Biol. Rev. 66, 300-372. doi: 10.1128/MMBR.66.2.300-372.2002

Jeon, J., Goh, J., Yoo, S., Chi, M. H., Choi, J., Rho, H. S., et al. (2008). A putative MAP kinase kinase kinase, MCK1, is required for cell wall integrity and pathogenicity of the rice blast fungus, Magnaporthe oryzae. Mol. PlantMicrobe Interact. 21, 525-534. doi: 10.1094/MPMI-21-5-0525

Jiang, C., Zhang, X., Liu, H., and Xu, J. R. (2018). Mitogen-activated protein kinase signaling in plant pathogenic fungi. PLoS Pathog. 14:e1006875. doi: 10.1371/journal.ppat.1006875

Jin, C., Kim, S. K., Willis, S. D., and Cooper, K. F. (2015). The MAPKKKs Ste11 and Bck1 jointly transduce the high oxidative stress signal through the cell wall integrity MAP kinase pathway. Microb. Cell 2, 329-342. doi: 10.15698/mic2015.09.226

Krijgsheld, P., Bleichrodt, R. V., Van Veluw, G. J., Wang, F., Müller, W. H., Dijksterhuis, J., et al. (2013). Development in Aspergillus. Stud. Mycol. 74, 1-29. doi: 10.3114/sim0006

Kuo, C. Y., Chen, S. A., and Hsueh, Y. P. (2020). The high osmolarity glycerol (HOG) pathway functions in osmosensing, trap morphogenesis and conidiation of the nematode-trapping fungus Arthrobotrys oligospora. J. Fungi 6:191. doi: $10.3390 /$ jof 6040191

Levdansky, E., Kashi, O., Sharon, H., Shadkchan, Y., and Osherov, N. (2010). The Aspergillus fumigatus cspA gene encoding a repeat-rich cell wall protein is important for normal conidial cell wall architecture and interaction with host cells. Eukaryot. Cell 9, 1403-1415. doi: 10.1128/EC.00126-10

Levin, D. E. (2005). Cell wall integrity signaling in Saccharomyces cerevisiae. Microbiol. Mol. Biol. Rev. 69, 262-291. doi: 10.1128/MMBR.69.2.262-291.2005

Levin, D. E. (2011). Regulation of cell wall biogenesis in Saccharomyces cerevisiae: the cell wall integrity signaling pathway. Genetics 189, 1145-1175. doi: 10.1534/genetics.111.128264

Liang, L., Gao, H., Li, J., Liu, L., Liu, Z., and Zhang, K. Q. (2017). The Woronin body in the nematophagous fungus Arthrobotrys oligospora is essential for trap formation and efficient pathogenesis. Fungal Biol. 121, 11-20. doi: 10.1016/j.funbio.2016.08.010

Liu, W., Soulié, M. C., Perrino, C., and Fillinger, S. (2011). The osmosensing signal transduction pathway from Botrytis cinerea regulates cell wall integrity and MAP kinase pathways control melanin biosynthesis with influence of light. Fungal Genet. Biol. 48, 377-387. doi: 10.1016/j.fgb.2010.12.004

Liu, J., Wang, Z., Sun, H., Ying, S., and Feng, M. (2017). Characterization of the Hog1 MAPK pathway in the entomopathogenic fungus Beauveria bassiana. Environ. Microbiol. 19, 1808-1821. doi: 10.1111/1462-2920.13671

Liu, K., Zhang, W., Lai, Y., Xiang, M., Wang, X., Zhang, X., et al. (2014). Drechslerella stenobrocha genome illustrates the mechanism of constricting rings and the origin of nematode predation in fungi. BMC Genomics 15:114. doi: 10.1186/1471-2164-15-114

Livak, K. J., and Schmittgen, T. D. (2001). Analysis of relative gene expression data using real-time quantitative PCR and the $2^{-\triangle \triangle C T}$ method. Methods 25, 402-408. doi: 10.1006/meth.2001.1262

Luo, X. D., Keyhani, N. O., Yu, X. D., He, Z. J., Luo, Z. B., Pei, Y., et al. (2012). The MAP kinase Bbslt2 controls growth, conidiation, cell wall integrity, and virulence in the insect pathogenic fungus Beauveria bassiana. Fungal Genet. Biol. 49, 544-555. doi: 10.1016/j.fgb.2012.05.002

Ma, Y., Yang, X., Xie, M., Zhang, G., Yang, L., Bai, N., et al. (2020). The Arf-GAP AoGlo3 regulates conidiation, endocytosis, and pathogenicity in the nematode-trapping fungus Arthrobotrys oligospora. Fungal Genet. Biol. 138:103352. doi: $10.1016 /$ j.fgb.2020.103352

Meerupati, T., Andersson, K. M., Friman, E., Kumar, D., Tunlid, A., and Ahrén, D. (2013). Genomic mechanisms accounting for the adaptation to parasitism in nematode-trapping fungi. PLoS Genet. 9:e1003909. doi: 10.1371/ journal.pgen.1003909

Mehrabi, R., van der Lee, T., Waalwijk, C., and Kema, G. H. (2006). MgSlt2, a cellular integrity MAP kinase gene of the fungal wheat pathogen Mycosphaerella graminicola, is dispensable for penetration but essential for invasive growth. Mol. Plant-Microbe Interact. 19, 389-398. doi: 10.1094/MPMI-19-0389

Momany, M., Richardson, E. A., Van Sickle, C., and Jedd, G. (2002). Mapping Woronin body position in Aspergillus nidulans. Mycologia 94, 260-266. doi: 10.1080/15572536.2003.11833231

Navarro-Espíndola, R., Suaste-Olmos, F., and Peraza-Reyes, L. (2020). Dynamic regulation of peroxisomes and mitochondria during fungal development. J. Fungi 6:302. doi: 10.3390/jof6040302
Nordbring-Hertz, B. (2004). Morphogenesis in the nematode-trapping fungus Arthrobotrys oligospora-an extensive plasticity of infection structures. Mycologist 18, 125-133. doi: 10.1017/S0269-915X (04) 00305-2

Nordbring-Hertz, B., Jansson, H. B., and Tunlid, A. (2001). "Nematophagous fungi” in Encyclopedia of Life Sciences. ed. O. H. Chichester (Hoboken, NJ: John Wiley \& Sons), 1-13.

Park, H. S., and Yu, J. H. (2012). Genetic control of asexual sporulation in filamentous fungi. Curr. Opin. Microbiol. 15, 669-677. doi: 10.1016/j.mib.2012.09.006

Su, H., Zhao, Y., Zhou, J., Feng, H., Jiang, D., Zhang, K. Q., et al. (2017). Trapping devices of nematode-trapping fungi: formation, evolution, and genomic perspectives. Biol. Rev. Camb. Philos. Soc. 92, 357-368. doi: 10.1111/ brv. 12233

Tunlid, A., Åhman, J., and Oliver, R. P. (1999). Transformation of the nematodetrapping fungus Arthrobotrys oligospora. FEMS Microbiol. Lett. 173, 111-116. doi: 10.1111/j.1574-6968.1999.tb13491.x

Tunlid, A., Jansson, H. B., and Nordbring-Hertz, B. (1992). Fungal attachment to nematodes. Mycol. Res. 96, 401-412. doi: 10.1016/S0953-7562 (09) 81082-4

Turrà, D., Segorbe, D., and Di, P. A. (2014). Protein kinases in plant-pathogenic fungi: conserved regulators of infection. Annu. Rev. Phytopathol. 52, 267-288. doi: 10.1146/annurev-phyto-102313-050143

Valiante, V., Jain, R., Heinekamp, T., and Brakhage, A. A. (2009). The MpkA MAP kinase module regulates cell wall integrity signaling and pyomelanin formation in Aspergillus fumigatus. Fungal Genet. Biol. 46, 909-918. doi: 10.1016/j.fgb.2009.08.005

Xie, M., Bai, N., Yang, J., Jiang, K., Zhou, D., Zhao, Y., et al. (2020). Protein kinase Ime2 is required for mycelial growth, conidiation, osmoregulation, and pathogenicity in nematode-trapping fungus Arthrobotrys oligospora. Front. Microbiol. 10:3065. doi: 10.3389/fmicb.2019.03065

Xie, M., Wang, Y., Tang, L., Yang, L., Zhou, D., Li, Q., et al. (2019). AoStuA, an APSES transcription factor, regulates the conidiation, trap formation, stress resistance and pathogenicity of the nematode-trapping fungus Arthrobotrys oligospora. Environ. Microbiol. 21, 4648-4661. doi: 10.1111/1462-2920.14785

Xu, J. R., Staiger, C. J., and Hamer, J. E. (1998). Inactivation of the mitogenactivated protein kinase Mps1 from the rice blast fungus prevents penetration of host cells but allows activation of plant defense responses. Proc. Natl. Acad. Sci. U. S. A. 95, 12713-12718. doi: 10.1073/pnas.95.21.12713

Yang, X., Ma, N., Yang, L., Zheng, Y., Zhen, Z., Li, Q., et al. (2018). Two Rab GTPases play different roles in conidiation, trap formation, stress resistance, and virulence in the nematode-trapping fungus Arthrobotrys oligospora. Appl. Microbiol. Biotechnol. 102, 4601-4613. doi: 10.1007/s00253-018-8929-1

Yang, C. T., Vidal-Diez De Ulzurrun, G., Gonçalves, A. P., Lin, H. C., Chang, C. W., Huang, T. Y., et al. (2020). Natural diversity in the predatory behavior facilitates the establishment of a robust model strain for nematode-trapping fungi. Proc. Natl. Acad. Sci. U. S. A. 117, 6762-6770. doi: 10.1073/pnas.1919726117

Yang, J., Wang, L., Ji, X., Feng, Y., Li, X., Zou, C., et al. (2011). Genomic and proteomic analyses of the fungus Arthrobotrys oligospora provide insights into nematode-trap formation. PLoS Pathog. 7:e1002179. doi: 10.1371/journal. ppat.1002179

Zarzov, P., Mazzoni, C., and Mann, C. (1996). The SLT2 (MPK1) MAP kinase is activated during periods of polarized cell growth in yeast. EMBO J. 15, 83-91. doi: 10.1002/j.1460-2075.1996.tb00336.x

Zhang, A. X., Mouhoumed, A. Z., Tong, S. M., Ying, S. H., and Feng, M. G. (2019a). BrlA and AbaA govern virulence-required dimorphic switch, conidiation, and pathogenicity in a fungal insect pathogen. mSystems 4, e00140-e00119. doi: 10.1128/mSystems.00140-19

Zhang, L., Wang, J., Xie, X., Keyhani, N. O., Feng, M., and Ying, S. (2013). The autophagy gene BbATG5, involved in the formation of the autophagosome, contributes to cell differentiation and growth but is dispensable for pathogenesis in the entomopathogenic fungus Beauveria bassiana. Microbiology 159, 243-252. doi: 10.1099/mic.0.062646-0

Zhang, G., Zheng, Y., Ma, Y., Yang, L., Xie, M., Zhou, D., et al. (2019b). The velvet proteins VosA and VelB play different roles in conidiation, trap formation, and pathogenicity in the nematode-trapping fungus Arthrobotrys oligospora. Front. Microbiol. 10:1917. doi: 10.3389/fmicb.2019.01917

Zhao, X., Kim, Y., Park, G., and Xu, J. R. (2005). A mitogen-activated protein kinase cascade regulating infection-related morphogenesis in Magnaporthe grisea. Plant Cell 17, 1317-1329. doi: 10.1105/tpc.104.029116

Zhen, Z., Xing, X., Xie, M., Yang, L., Yang, X., Zheng, Y., et al. (2018). MAP kinase Slt2 orthologs play similar roles in conidiation, trapformation, 
and pathogenicity in two nematode-trapping fungi. Fungal Genet. Biol. 116, 42-50. doi: $10.1016 /$ j.fgb.2018.04.011

Conflict of Interest: The authors declare that the research was conducted in the absence of any commercial or financial relationships that could be construed as a potential conflict of interest.
Copyright () 2021 Xie, Yang, Jiang, Bai, Zhu, Zhu, Zhang and Yang. This is an openaccess article distributed under the terms of the Creative Commons Attribution License (CC BY). The use, distribution or reproduction in other forums is permitted, provided the original author(s) and the copyright owner(s) are credited and that the original publication in this journal is cited, in accordance with accepted academic practice. No use, distribution or reproduction is permitted which does not comply with these terms. 